Zbl Arbeitsmed 2022 $\cdot 72: 13-19$ https://doi.org/10.1007/s40664-021-00445-7 Eingegangen: 26. Februar 2021 Angenommen: 25. August 2021 Online publiziert: 29 . September 2021 c c Der/die Autor(en) 2021

\section{Zusatzmaterial online}

Zusätzliche Informationen sind in der Online-Version dieses Artikels (https://doi. org/10.1007/s40664-021-00445-7) enthalten.

Muskel-Skelett-Erkrankungen (MSE) sind besonders oft in Berufen mit hoher körperlicher Beanspruchung anzutreffen. Friseur*innen üben häufig Tätigkeiten aus, die mit strukturbelastenden Körperhaltungen und Bewegungen einhergehen. MSE sind nach Haut- und Atemwegserkrankungen der dritthäufigste Grund für Verdachtsanzeigen von Berufskrankheiten im Friseurhandwerk [12]. Daten von Krankenversicherungen zeigen, dass ein Fünftel der Fehlzeiten auf MSE zurückzuführen sind. In diesem Beitrag stellen wir die Ergebnisse einer Prävalenzstudie zu Muskel-Skelett-Beschwerden (MSB) bei Friseur*innen vor.

Beobachtungsstudien und messtechnische Analysen haben gezeigt, dass bei der täglichen Arbeit von Friseur*innen häufig ungünstige Körperhaltungen und Gelenkwinkelstellungen entstehen [12, 13, 22]. Besonders betroffen sind u. a. die Schultergelenke, wenn z. B. beim Schneiden, Färben und Föhnen die Arme bis über das Schulterniveau angehoben werden. Auch in allen Bereichen der Wirbelsäule (Nacken, oberer und unterer

Michaela Wolff ${ }^{1}$ Dania Kitzig ${ }^{2}$ · Sonja Freitag ${ }^{1}$. Agnessa Kozak ${ }^{2}$. Albert Nienhaus ${ }^{1,2}$

${ }^{1}$ Hauptverwaltung, Berufsgenossenschaft für Gesundheitsdienst und Wohlfahrtspflege, Hamburg, Deutschland

${ }^{2}$ Universitätsklinikum Hamburg-Eppendorf, Institut für Versorgungsforschung in der Dermatologie und bei Pflegeberufen (IVDP), Kompetenzzentrum für Epidemiologie und Versorgungsforschung bei Pflegeberufen (CVcare), Hamburg, Deutschland

\title{
Muskuloskeletale Beschwerden bei Friseur*innen
}

\section{Eine Querschnittsstudie zur 12-Monate- Prävalenz in Deutschland}

Rücken) wurden ungünstige Gelenkwinkelstellungen erfasst: So kommt es beim Waschen und Schneiden der Haare oftmals $\mathrm{zu}$ einer Verschiebung der Halswirbelsäule nach vorne, kombiniert mit einer gleichzeitigen Überstreckung nach hinten und einer Krümmung der Brustwirbelsäule [12, 13]. Zusätzliche physische Belastungen entstehen durch statische Körperhaltungen insbesondere im Bereich des oberen Rückens und durch repetitive Bewegungen, die am häufigsten an den Schulter-, Hand- und Fingergelenken auftreten $[12,13]$. Längsstudien mit Auszubildenden im Friseurhandwerk zeigen, dass MSB schon während der Ausbildung auftreten können [9-11].

Die höchsten 12-Monate-Prävalenzraten von arbeitsbedingten MSB weisen einem Scoping Review von Kozak et al. zufolge die Körperregionen unterer Rücken, Nacken, Schultern und Hände/Handgelenke auf. Die internationalen Forschungsergebnisse $\mathrm{zu}$ der Prävalenz von MSB im Friseurhandwerk sind jedoch sehr heterogen [14] und für Deutschland liegen bisher keine Daten zur Prävalenz verschiedener Körperregionen vor.

Die Berufsgenossenschaft für $\mathrm{Ge}$ sundheitsdienst und Wohlfahrtspflege (BGW) initiierte in Kooperation mit dem Universitätsklinikum Hamburg-Eppendorf und dem Institut für Arbeitsschutz der Deutschen Gesetzlichen Unfallver- sicherung ein mehrteiliges Forschungsprojekt mit dem Ziel, die Ursachen für MSB bei Friseur ${ }^{\star}$ innen zu untersuchen, um anschließend geeignete Präventionsmaßnahmen abzuleiten. In Stufe 1 wurde mittels Krankenversicherungsdaten der durch MSE bedingte Krankenstand bei Friseur*innen ermittelt. Zudem haben wir mithilfe der Videoanalyse von Arbeitsschichten Tätigkeitsprofile erstellt und strukturbelastende Körperhaltungen und Bewegungen identifiziert [12]. In Stufe 2 wurden in einer Machbarkeitsstudie Köperhaltungen und Bewegungen bei typischen Friseurtätigkeiten messtechnisch analysiert [13]. In diesem Artikel berichten wir über den dritten Teil des Projekts, der im Rahmen einer Querschnittsstudie folgende Forschungsfragen untersuchte:

1. Wie hoch ist die 12-Monate-Prävalenz von MSB und beruflicher Beeinträchtigung in verschiedenen

\begin{tabular}{|ll}
\hline Abkürzungen \\
\hline$B G W$ & $\begin{array}{l}\text { Berufsgenossenschaft für Gesund- } \\
\text { heitsdienst und Wohlfahrtspflege }\end{array}$ \\
\hline$B M I$ & Body-Mass-Index \\
\hline$M S B$ & Muskel-Skelett-Beschwerden \\
\hline$M S E$ & Muskel-Skelett-Erkrankungen \\
\hline$R$ & Range (Spannweite) \\
\hline$S N Q$ & $\begin{array}{l}\text { Standardised Nordic Question- } \\
\text { naire }\end{array}$ \\
\hline
\end{tabular}


Friseurbetriebe bei der BGW ( $N=82.319)$

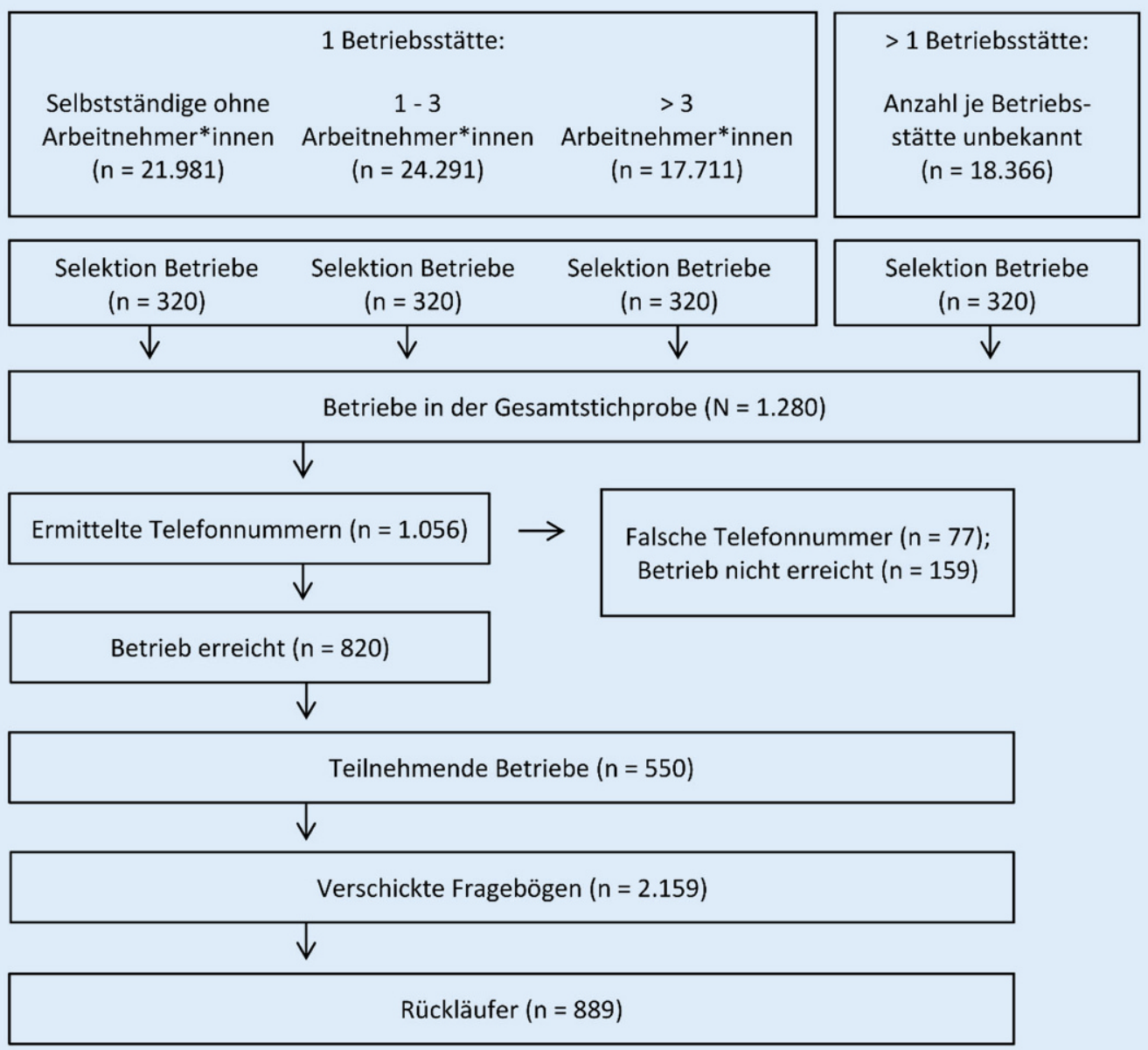

Abb. $1<$ Stichprobenselektion aus der Grundgesamtheit der Mitgliedsbetriebe der Berufsgenossenschaft für Gesundheitsdienst und Wohlfahrtspflege (BGW)

Körperregionen bei Friseur*innen in Deutschland?

2. Wie häufig kommen schwere MSB (an mehr als 30 Tagen im Jahr mit beruflicher Beeinträchtigung) vor?

3. Unterscheiden sich Friseur*innen, die über MSB oder schwere MSB berichten, und solche, die aufgrund von MSB ihre wöchentliche Arbeitszeit reduziert haben, hinsichtlich ihrer Charakteristika?

\section{Methode}

\section{Studienpopulation}

Um sicherzustellen, dass aus Friseurbetrieben mit einer bzw. mehr als einer Betriebsstätte (mit und ohne Arbeitnehmer*innen) jeweils genügend $\mathrm{Be}$ triebe ausgewählt werden, haben wir eine stratifizierte Stichprobenauswahl mit 4 Gruppen durchgeführt. Aus jeder Gruppe wurde eine Zufallsauswahl von 320 Betrieben gezogen (• Abb. 1). Die Betriebe wurden telefonisch kontaktiert, und bei einer Einwilligung zur Teilnahme wurden Papierfragebögen versendet. Einbezogen wurden praktizierende Friseur*innen mit direktem Kundenkontakt.

\section{Messinstrument}

Für die Erhebung von MSB wurde der Standardised Nordic Questionnaire (SNQ) in einer adaptierten Version eingesetzt. Als Grundlage diente die Übersetzung von Caffier [2], und die grafische Gestaltung wurde der Zielgruppe entsprechend angepasst. Mit dem SNQ werden selbstberichtete MSB in den vergangenen 12 Monaten an verschiedenen Körperregionen erfasst [15]. In dieser Studie wurden MSB an den folgenden 11 Körperregionen erfragt: Nacken, oberer und unterer Rücken, Schultern, Ellenbogen, Handgelenke, Daumen, Finger, Hüfte, Knie sowie Füße. Das Vorliegen von MSB konnte nach der Häufigkeit der Beschwerden mit „Ja, an 1 bis 7 Tagen“, „an 8 bis 30 Tagen“, „an mehr als 30 Tagen“ oder „täglich“ angegeben werden. Auch wurde erhoben, ob die Beschwerden die Friseur*innen während ihrer beruflichen Tätigkeit beeinträchtigen. Die Teilnehmer*innen wurden außerdem gefragt, ob die Wochenarbeitszeit jemals wegen MSB reduziert wurde. Basierend auf den Vorstudien [12, 13] wurde eine Auswahl von demografischen (Alter, Geschlecht), anthropometrischen (Gewicht 
und Größe) und berufsbezogenen Variablen erhoben (Berufsfeld, Ausbildungs-, bzw. Berufsstatus, Spezialisierung, Dauer der Berufserfahrung sowie die Anzahl der Arbeitsstunden pro Woche im und außerhalb des Salons). Ein Pretest des Fragebogens wurde in 9 Friseursalons mit 42 Friseur*innen durchgeführt.

\section{Statistische Analyse}

Das Hauptaugenmerk lag auf der Analyse der 12-Monate-Prävalenz von MSB in verschiedenen Körperregionen, wobei es sich hierbei um die MSB in den zurückliegenden 12 Monaten vor Beantwortung des Fragebogens handelt. Basierend auf der Häufigkeit von MSB und einer beruflichen Beeinträchtigung wurden zudem „schwere MSB“ ermittelt. Es handelt sich dabei um Beschwerden an mehr als 30 Tagen im Jahr und von denen sich Betroffene beruflich beeinträchtigt fühlen. Kontinuierliche Variablen haben wir gruppiert und die Körperregionen für die Analyse schwerer MSB wie folgt zusammengefasst: Wirbelsäule und Schultern (Nacken, oberer Rücken, unterer Rücken und Schultern), obere Extremitäten (Ellenbogen, Handgelenk, Daumen und Finger) und untere Extremitäten (Hüfte, Knie und Füße). Die berufliche Stellung wurde nach „selbstständig" und „angestellt" dichotomisiert. Die Analyse der erhobenen Daten erfolgte deskriptiv und interferenzstatistisch. Die deskriptive Statistik umfasste absolute Häufigkeiten und gültige Prozente. Für kontinuierliche Variablen wurden zusätzlich Mittelwerte und Standartabweichungen berechnet. Unterschiede der Prävalenz von schweren MSB und jemals reduzierter Wochenarbeitszeit wurden mit dem $\mathrm{Chi}^{2}$-Test bzw. für kontinuierliche Variablen mit dem Mann-WhitneyU-Test auf einem Signifikanzniveau von $p<0,05$ untersucht. Die Effektstärken der $\mathrm{Chi}^{2}$-Tests wurden mit Cramers V und die der Mann-Whitney-U-Tests mit der Formel $\mathrm{r}=\mathrm{z} /(\sqrt{ } n)$ berechnet und nach Cohen [3] interpretiert (Cramers $\mathrm{V}=0,1$ schwacher, $\mathrm{V}=0,3$ mittlerer, $\mathrm{V}=0,5$ starker Zusammenhang). Alle Berechnungen wurden mit PASW Statistics 18 (SPSS Inc., Chicago, IL, USA) durchgeführt.

Zbl Arbeitsmed 2022 · 72:13-19 https://doi.org/10.1007/s40664-021-00445-7

(c) Der/die Autor(en) 2021

M. Wolff · D. Kitzig · S. Freitag · A. Kozak · A. Nienhaus

Muskuloskeletale Beschwerden bei Friseur*innen. Eine
Querschnittsstudie zur 12-Monate-Prävalenz in Deutschland

Zusammenfassung

Hintergrund. Friseur*innen arbeiten häufig in strukturbelastenden Körperhaltungen und haben ein erhöhtes Risiko für arbeitsbedingte Muskel-Skelett-Beschwerden (MSB). MSB verursachen in Deutschland circa ein Fünftel der Arbeitsunfähigkeitstage im Friseurhandwerk. Um das Ausmaß der Verbreitung von MSB bei Friseur*innen in Deutschland zu bestimmen, wurde eine Prävalenzstudie durchgeführt. Methode. Die Studie war eine bundesweite Befragung im Querschnittsdesign, woran 889 Friseur*innen teilnahmen (Rücklauf $41 \%$ ). Die 12-Monate-Prävalenz von MSB wurde mit dem Standardised Nordic Questionnaire erfasst. Die Analyse der erhobenen Daten erfolgte deskriptiv und interferenzstatistisch. Ergebnisse. Insgesamt $91 \%$ der Befragten hatten in mindestens einer Körperregion MSB. Die am häufigsten von MSB bzw. schweren MSB (mind. 30 Tage/Jahr und beruflich beeinträchtigt) betroffenen Körperregionen sind Nacken (70\%; $25 \%)$, unterer Rücken
(65\%; $25 \%)$, Schultern (61\%; $23 \%)$ und oberer Rücken (58\%; $22 \%$ ). Beschwerden in diesen Bereichen führten auch am häufigsten zu beruflichen Beeinträchtigungen. Faktoren wie das weibliche Geschlecht, ein hohes Alter, Adipositas, viele Berufsjahre und Selbstständigkeit sind mit schweren MSB assoziiert.

Schlussfolgerung. Diese Studie liefert erste Daten für die Prävalenz von MSB in verschiedenen Körperregionen bei Friseur*innen in Deutschland. Die Ergebnisse weisen auf eine hohe Belastung des MuskelSkelett-Systems bei Friseur*innen hin. Daraus resultiert ein hoher Bedarf an Prävention von arbeitsbedingten MSB im Friseurhandwerk.

\section{Schlüsselwörter}

Muskel-Skelett-Beschwerden · Berufliche Beeinträchtigung $\cdot$ Friseurhandwerk · Standardised Nordic Questionnaire · Gesundheit am Arbeitsplatzs

\section{Musculoskeletal symptoms in hairdressers. A cross-sectional study on 12-month prevalence in Germany}

\section{Abstract}

Background. Hairdressers often work in postures that are structurally stressful and have an increased risk of work-related musculoskeletal symptoms (MSS). In Germany, MSS cause about one fifth of the days of incapacity to work in the hairdressing trade. In order to determine the extent of the prevalence of MSS among hairdressers in Germany, a prevalence study was conducted. Method. The study was a nationwide survey with a cross-sectional design, in which 889 hairdressers participated (response rate 41\%). The 12-month prevalence of MSS was assessed with the Standardized Nordic Questionnaire. The collected data were analyzed descriptively and interferentially. Results. Of the respondents $91 \%$ had MSS in at least 1 body region. The body regions most frequently affected by MSS or severe MSS (at least 30 days/year and occupationally impaired) were the neck (70\%; $25 \%)$, lower back (65\%; $25 \%)$, shoulders (61\%; $23 \%$ ) and upper back $(58 \% ; 22 \%)$. Complaints in these areas also most frequently led to occupational impairment. Factors such as female gender, advanced age, obesity, many years of work and self-employment are associated with severe MSS.

Conclusion. This study provides the first data on the prevalence of MSS in various body regions among hairdressers in Germany. The results indicate a high burden on the musculoskeletal system among hairdressers. This results in a high need for prevention of work-related MSS in the hairdressing sector.

\section{Keywords}

Musculoskeletal pain - Occupational impairment $\cdot$ Hairdressing trade $\cdot$ Standardised Nordic Questionnaire · Occupational health 


\section{Originalien}

\begin{tabular}{|c|c|c|}
\hline Variable & & $n(\%)$ \\
\hline \multirow[t]{2}{*}{ Geschlecht } & Weiblich & $805(90,6)$ \\
\hline & Männlich & $84(9,4)$ \\
\hline \multirow[t]{4}{*}{ Alter } & Unter 25 Jahre & $168(18,9)$ \\
\hline & 25 bis unter 35 Jahre & $210(23,7)$ \\
\hline & 35 bis unter 50 Jahre & $273(30,8)$ \\
\hline & 50 Jahre und älter & $236(26,6)$ \\
\hline \multirow{4}{*}{$\begin{array}{l}\text { BMI (Klassifikation nach WHO } 2000 \\
\text { [23]) }\end{array}$} & Untergewicht & $40(4,6)$ \\
\hline & Normalgewicht & $518(59,3)$ \\
\hline & Übergewicht & $224(25,7)$ \\
\hline & Adipositas & $91(10,4)$ \\
\hline \multirow[t]{4}{*}{ Beruflicher Ausbildungsstand } & Ohne Ausbildung & $17(2,0)$ \\
\hline & Auszubildende*r & $81(9,3)$ \\
\hline & Geselle*in & $463(53,2)$ \\
\hline & Meister*in & $310(35,6)$ \\
\hline \multirow[t]{3}{*}{ Berufliche Stellung } & Angestellt & $616(69,8)$ \\
\hline & Selbstständig & $247(28,0)$ \\
\hline & Angestellt und selbständig & $19(2,2)$ \\
\hline \multirow[t]{3}{*}{ Fachbereich der Tätigkeit } & Damenfach & $44(5,0)$ \\
\hline & Herrenfach & $23(2,6)$ \\
\hline & Damen- und Herrenfach gemischt & $811(92,4)$ \\
\hline \multirow[t]{5}{*}{ Berufsjahre } & Unter 10 Jahre & $234(26,8)$ \\
\hline & 10 bis unter 20 Jahre & $181(20,7)$ \\
\hline & 20 bis unter 30 Jahre & $160(18,3)$ \\
\hline & 30 bis unter 40 Jahre & $177(20,3)$ \\
\hline & 40 Jahre und mehr & $122(14,0)$ \\
\hline \multirow[t]{4}{*}{ Wochenarbeitszeit } & Unter $20 \mathrm{~h}$ & $116(13,3)$ \\
\hline & 20 bis unter $38 \mathrm{~h}$ & $298(34,2)$ \\
\hline & 38 bis $40 h$ & $250(28,7)$ \\
\hline & Über 40h & $208(23,9)$ \\
\hline \multirow{2}{*}{$\begin{array}{l}\text { Jemals Wochenarbeitszeit wegen MSB } \\
\text { reduziert }\end{array}$} & Ja & $119(13,4)$ \\
\hline & Nein & $767(86,6)$ \\
\hline
\end{tabular}

\section{Ergebnisse}

\section{Stichprobenbeschreibung}

Insgesamt haben 889 Friseur ${ }^{\star}$ innen aus allen Bundesländern den Fragebogen beantwortet (Rücklaufquote 40,8\%). - Tab. 1 zeigt die Charakteristika der Stichprobe. Ein Großteil (90,6\%) der Beschäftigten ist weiblich und durchschnittlich $39( \pm 13,7)$ Jahre alt. Im Mittel haben die Befragten einen BMI von 24,3 $( \pm 4,4), 22,1( \pm 14,4)$ Jahre Berufserfahrung und eine Wochenarbeitszeit von $33,7( \pm 11,7)$ h. Mehr als ein Drittel der Friseur*innen hat einen Meistertitel (35,6\%), und ein knappes Drittel ist ausschließlich selbstständig tätig (28\%). ben als solche an den oberen und unteren Extremitäten (40-47\% vs. 10-25\%).

\section{Schwere MSB}

Insgesamt $46,5 \% \quad(n=369) \quad$ der Friseur*innen berichten in mindestens einer der untersuchten Körperregionen von schweren MSB (an mehr als $30 \mathrm{Ta}$ gen und beruflich beeinträchtigt). Dabei traten schwere MSB besonders häufig an der Wirbelsäule (Nacken, oberer und unterer Rücken) und den Schultern auf (- Tab. 2). Hier sind 41,5\% $(n=344)$ aller Friseur*innen von schweren MSB in mindestens einer dieser Körperregionen und $8,9 \%(n=74)$ in allen 4 Regionen betroffen. An den oberen und unteren Extremitäten ist die Prävalenz schwerer MSB hingegen deutlich geringer (15,6\% bzw. 21,9\%).

Frauen berichteten signifikant häufiger von schweren MSB an den Körperregionen Nacken, oberer Rücken und unterer Rücken als Männer $(p<0,05)$. Beschäftigte mit schweren MSB in den unteren Extremitäten hatten einen höheren BMI als solche ohne Beschwerden $(p<0,01)$. Die von schweren MSB in allen Körperregionen Betroffenen waren im Durchschnitt älter und hatten mehr Berufsjahre als diejenigen ohne Beschwerden (jeweils $p<0,01$ ). Selbstständige berichten im Vergleich zu Angestellten häufiger über schwere Schulterbeschwerden $(p<0,05)$. Alle ermittelten Effekte sind mit einem Cramers V bzw. $\mathrm{r}<0,3(p<0,05)$ schwach (Zusatzmaterial online: Appendix Unterschiedstests).

\section{Jemals reduzierte Wochenarbeits- zeit wegen MSB}

gangenen 12 Monaten sowie die 12Monate-Prävalenz von MSB und beruflicher Beeinträchtigung sind der • Abb. 2 zu entnehmen. 85,8\% $(n=763)$ der Teilnehmer*innen berichten von MSB in mindestens einer Körperregion in den vergangenen 12 Monaten (Daten nicht gezeigt). Am häufigsten traten MSB in den Körperregionen Nacken (70,3\%; $n=619)$, unterer Rücken $(65,4 \%$; $n=573)$, Schultern $(61,2 \% ; n=541)$ und oberer Rücken $(57,6 \% ; n=502)$ auf. In diesen Bereichen wurden auch häufiger berufliche Beeinträchtigungen angege-
Friseur*innen, die jemals wegen MSB ihre Arbeitsstunden reduziert haben $(13,4 \%)$, sind im Vergleich zu denjenigen, die dies nicht angeben, signifikant häufiger von schweren MSB an allen Körperregionen betroffen $(p<0,001)$. sind mit einer jemals reduzierten Wochenarbeitszeit wegen MSB assoziiert $(p<0,001)$. Selbstständige, Gesell ${ }^{*}$ innen und Meister*innen geben ebenfalls häufiger an, ihre Wochenarbeitszeit wegen MSB reduziert zu haben $(p<0,001)$. Die Ein hohes Alter und viele Berufsjahre 


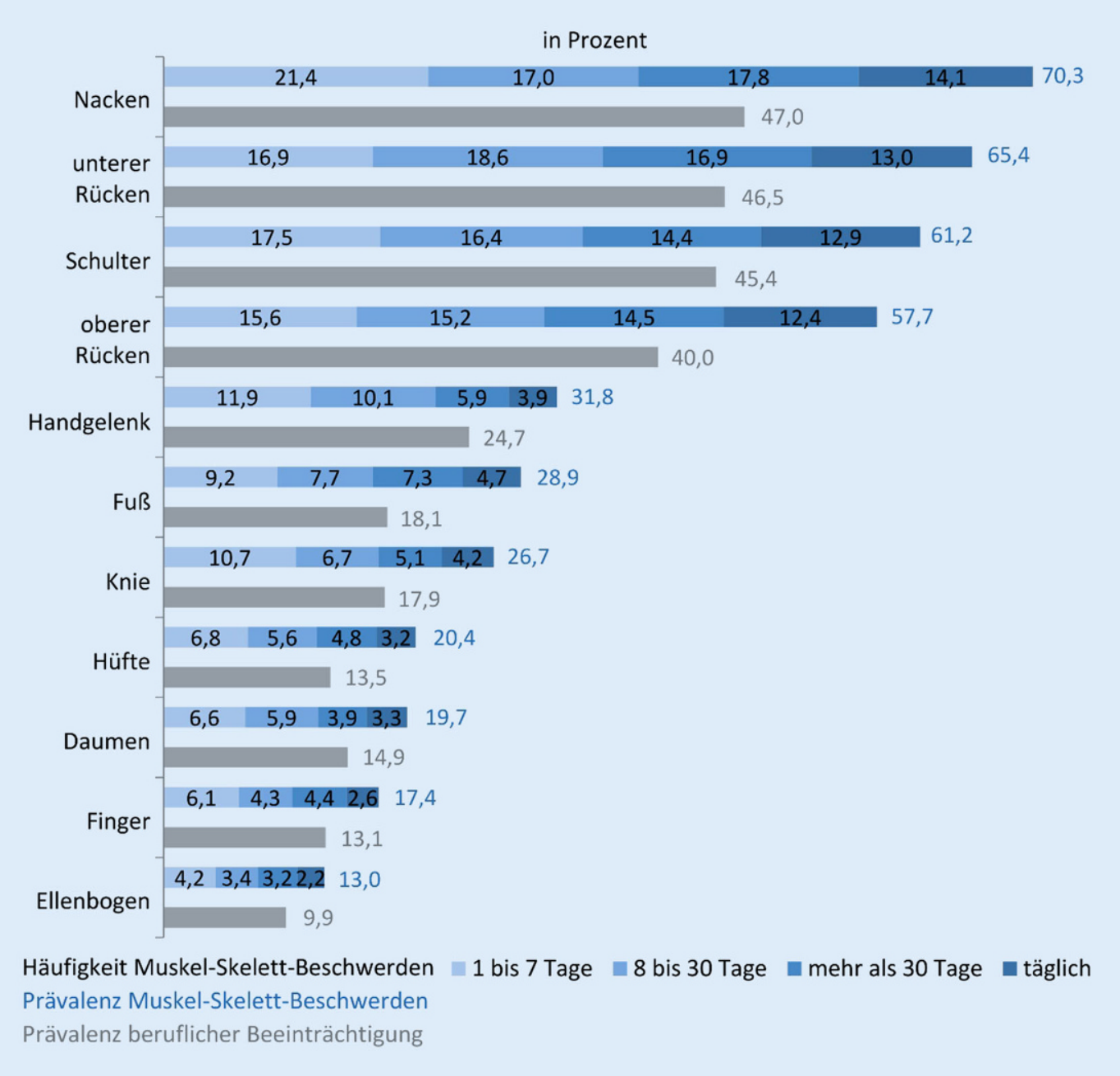

Abb. $2 \triangleleft$ Häufigkeit von Muskel-Skelett-Beschwerden (MSB) in den vergangenen 12 Monaten sowie 12Monate-Prävalenz von MSB und beruflicher Beeinträchtigung, $n$ variiert zwischen 858 und 884. Rundungsfehler

ermittelten Effektstärken liegen unter 0,3 und sind schwach (Zusatzmaterial online: Appendix Unterschiedstests).

\section{Diskussion}

Die vorliegende Studie ist die erste bundesweite Prävalenzstudie zu MSB in verschiedenen Körperregionen bei Friseur*innen in Deutschland. Zudem werden erstmals Charakteristika von Friseur*innen mit schweren MSB sowie von Friseur ${ }^{\star}$ innen, die ihre wöchentliche Arbeitszeit aufgrund von MSB reduziert haben, berichtet.

Die Ergebnisse zeigen, dass ein Großteil der befragten Friseur*innen (86\%) in mindestens einer der untersuchten Körperregionen MSB hatte. Die Körperregionen Nacken, unterer Rücken, Schultern und oberer Rücken sind am häufigsten betroffen, und etwa zwei Fünftel der Befragten (42\%) fühlten sich von diesen Beschwerden bei der beruflichen Tätigkeit beeinträchtigt. Etwa die Hälfte (47\%) berichtet sogar über schwere MSB, und $13 \%$ der Friseur*innen geben an, dass sie im Laufe ihres Berufslebens wegen MSB ihre wöchentliche Arbeitszeit reduziert hätten.

\section{Prävalenz von MSB}

Die hohe Prävalenz von MSB in den Körperregionen Nacken, oberer und unterer Rücken sowie Schultern deuten auf eine hohe Belastung dieser Körperregionen hin. Analog dazu beobachten Kitzig et al. $[12,13]$ in den ersten Studien dieses Forschungsprojektes v. a. am Rücken und den Schultern Körperhaltungen und Bewegungen, die mit ungünstigen Gelenkwinkelstellungen, Repetitionen und statischen Phasen einhergehen. Insgesamt sind Friseur*innen, verglichen mit Beschäftigten in anderen Berufsgruppen, überdurchschnittlich häufig von MSB betroffen. In einer repräsentativen bundesweiten Studie wurde für verschiedene Berufe die Prävalenz von Rückenschmerzen analysiert, dabei belegte die 12-MonatePrävalenz der Friseur*innen und Kosmetiker ${ }^{\star}$ innen den 5 . Rang (69,7\%; [21]). Einem britischen nationalen Gesundheitssurvey zufolge sind Friseur*innen unter den Top-6-Berufen mit einem erhöhten Risiko für Rückenschmerzen [8] und einer Auswertung französischer Surveillance-Daten zufolge unter den Top-10Berufen mit einem erhöhten Risiko für ein Karpaltunnelsyndrom [20].

Allerdings ist im Vergleich mit anderen europäischen Prävalenzstudien im Friseurhandwerk die in dieser Studie ermittelte Prävalenz von MSB in den Körperregionen Schultern (61\% vs. 


\begin{tabular}{|c|c|c|c|c|c|c|}
\hline $\begin{array}{l}\text { Gruppierte } \\
\text { Körperregion }\end{array}$ & Körperregion & $\begin{array}{l}\text { Schwere MSB } \\
n(\%)\end{array}$ & $\begin{array}{l}\text { In min. } 1 \text { Region } \\
n(\%)\end{array}$ & $\begin{array}{l}\text { In min. } 2 \text { Regionen } \\
n(\%)\end{array}$ & $\begin{array}{l}\text { In min. } 3 \text { Regionen } \\
n(\%)\end{array}$ & $\begin{array}{l}\text { In allen } 4 \text { Regionen } \\
n(\%)\end{array}$ \\
\hline \multirow{4}{*}{$\begin{array}{l}\text { Wirbelsäule und } \\
\text { Schulter }\end{array}$} & Nacken & $215(24,8)$ & \multirow[t]{4}{*}{$344(41,5)$} & \multirow[t]{4}{*}{$224(27,0)$} & \multirow[t]{4}{*}{$140(16,9)$} & \multirow[t]{4}{*}{$74(8,9)$} \\
\hline & Oberer Rücken & $187(21,9)$ & & & & \\
\hline & Unterer Rücken & $213(24,7)$ & & & & \\
\hline & Schulter & $199(22,9)$ & & & & \\
\hline \multirow[t]{4}{*}{ Obere Extremitäten } & Ellenbogen & $39(4,5)$ & \multirow[t]{4}{*}{$133(15,6)$} & \multirow[t]{4}{*}{$45(5,3)$} & \multirow[t]{4}{*}{$20(2,3)$} & \multirow[t]{4}{*}{$6(0,7)$} \\
\hline & Handgelenk & $75(8,6)$ & & & & \\
\hline & Finger & $52(5,9)$ & & & & \\
\hline & Daumen & $49(5,6)$ & & & & \\
\hline \multirow{3}{*}{$\begin{array}{l}\text { Untere Extremitä- } \\
\text { ten }\end{array}$} & Hüfte & $54(6,2)$ & \multirow[t]{3}{*}{$187(21,9)$} & \multirow[t]{3}{*}{$40(4,7)$} & \multirow[t]{3}{*}{$13(1,5)$} & \multirow[t]{3}{*}{-} \\
\hline & Knie & $60(6,9)$ & & & & \\
\hline & Fuß & $76(8,8)$ & & & & \\
\hline
\end{tabular}

$\mathrm{R}=28-53 \%[1,6,22])$, Nacken (70\% vs. $\mathrm{R}=37-52 \%[1,6,22])$, unterer Rücken (65\% vs. $\mathrm{R}=34-70[1,6,21])$ und oberer Rücken (58\% vs. $36 \%$ [1]) eher hoch. Eine mögliche Erklärung dafür sind die Unterschiede in der Studienpopulation. Die Stichproben der Studien mit niedrigerer Prävalenz wiesen ein jüngeres Durchschnittsalter (39 Jahre vs. $\mathrm{R}=27-32$ Jahre $[1,6,22])$ und einen höheren Anteil von Auszubildenden (9\% vs. $\mathrm{R}=19-23 \%[1,6]$ ) auf. Die Höhe der in dieser Studie ermittelten Prävalenz könnte zudem auf einen Selektionsbias zurückzuführen sein, da in dieser Studienpopulation mehr ältere Friseur*innen sind als unter den sozialversicherungspflichtigen Beschäftigten im Friseurhandwerk in Deutschland: Der Anteil der mindestens 50-Jährigen ist in der Stichprobe fast doppelt so hoch (26,6\% vs. $14,8 \%$ ), der Anteil der unter 35 -Jährigen hingegen niedriger (23,5\% vs. $34,8 \%$, [12]).

\section{Berufliche Beeinträchtigung durch MSB}

In diesem Setting sind uns bisher keine Studien bekannt, die schwere MSB sowie eine jemals reduzierte Wochenarbeitszeit wegen MSB untersucht haben. Die Relevanz der Studienergebnisse begründet sich darin, dass MSB langfristig zur Aufgabe des Berufs führen können. Zwei dänischen Studien zufolge sind muskuloskeletale Schmerzen die häufigste Ursache zum Aufgeben des Berufs bei
Friseur*innen mit gesundheitlichen Beschwerden [7, 17]. Eine weitere Studie aus Finnland berechnete bei Friseur*innen mit MSE ein erhöhtes Risiko, aus dem Beruf auszusteigen [16].

\section{Mit MSB assoziierte Faktoren}

Andere Studien zu Risikofaktoren für MSB bestätigen zudem das weibliche Geschlecht [4] und die Berufsjahre [18, 19] als Risikofaktoren für MSB bei Friseur*innen. Laut Hanvold et al. [9-11] können schon in den ersten Berufsjahren Schmerzen im Nacken und in den Schultern auftreten. Konträr zu unseren Studienergebnissen berichten Deschamps et al. [5], dass in arbeitsmedizinischen Untersuchungen selbstständig tätige Friseur*innen häufiger über MSB klagen als Angestellte. Sie begründen dies damit, dass Selbstständige zum Teil eine deutlich längere Wochenarbeitszeit haben als angestellte Friseur*innen.

\section{Limitationen}

Eine Limitation ergibt sich daraus, dass aufgrund methodischer und organisatorischer Aspekte Non-Responder nicht in der nötigen Tiefe analysiert werden konnten. Die Repräsentativität könnte weiterhin durch die abweichende Altersstruktur der Studienpopulation und durch eine Überrepräsentation von Beschäftigten aus Betrieben mit mindestens drei Beschäftigten verringert sein. Außerdem wurden $\mathrm{zu}$ wenige Informationen über verhältnis- und verhaltenspräventive Maßnahmen erhoben. Durch die retrospektive Erhebung der MSB könnten die Ergebnisse von einem RecallBias beeinflusst werden. Ein erhöhtes Mitteilungsbedürfnis von Personen mit Beschwerden kann dazu führen, dass vermehrt Friseur*innen mit MSB an der Befragung teilnahmen, was einen Responder-Bias zur Folge haben könnte. Eine weitere Limitation ist die fehlende Kontrollgruppe. Bei der Interpretation der Ergebnisse ist auch zu beachten, dass Querschnittsstudien keinen Aufschluss über die Kausalität von Beziehungen geben können.

\section{Ausblick}

Die hohe Beanspruchung der Friseur*innen durch MSB bestätigt die Beobachtungen aus unseren vorangegangenen Tätigkeits- und Körperhaltungsanalysen [12, 13]. Die beiden Studien zeigten, dass körperliche Belastungen an der Wirbelsäule und den oberen Extremitäten überwiegend durch eine Vielzahl strukturbelastender Körperhaltungen und Bewegungen hervorgerufen werden. Diese könnten sich jedoch durch alternative und schonende Bewegungsabläufe sowie Verhaltensweisen vermeiden lassen. Da MSB auch bei Berufsanfängern beobachtet werden, besteht ein möglicher Ansatz darin, die Friseur*innen bereits früh in der Ausbildung für ergonomische Arbeitsweisen und die Auswahl von ergonomischem 
Equipment zu sensibilisieren. Ein weiterer Fokus sollte auf selbstständige Friseur*innen gerichtet werden, da sie häufiger von MSB betroffen sind.

Trotz der dargestellten großen Beanspruchung sind prospektive Längsschnittstudien mit Kontrollgruppendesign notwendig, um den Kausalzusammenhang von beruflichen Belastungen im Friseurhandwerk und MSB zu bestätigen. Außerdem gibt es in diesem Setting bisher nur wenige Interventionsstudien, und die vorhandenen zeigen keine eindeutigen Ergebnisse. Daher sind hier weitere vergleichende Studien mit hoher methodischer Qualität erforderlich, um präventive Strategien zur Vorbeugung von MSB bei Friseur*innen auf der Verhaltens- und Verhältnisebene zu kombinieren.

\section{Fazit für die Praxis}

\section{- Die Studienergebnisse zeigen ei- ne hohe Beanspruchung der Fri- seur*innen, da ein Großteil der Friseur*innen in mindestens einer Körperregion MSB angibt. \\ - Aus der beruflichen Beeinträch- tigung bei knapp der Hälfte der Friseur*innen resultiert ein hoher Bedarf an effektiven Präventions- maßnahmen. \\ - Die hohe Prävalenz von MSB bei Be- schäftigten mit vielen Berufsjahren erfordert eine adäquate arbeitsme- dizinische Versorgung und Rehabi- litation, um die Arbeitsfähigkeit zu erhalten. \\ - Bei Präventions- und Rehabilitations- maßnahmen sollte ein besonderes Augenmerk auf die Bereiche der Wirbelsäule und der Schultern ge- legt werden, da diese am häufigsten von MSB betroffenen sind und dort MSB häufig in mehreren Bereichen gleichzeitig auftreten.}

\section{Korrespondenzadresse}

\section{Michaela Wolff}

Hauptverwaltung, Berufsgenossenschaft für Gesundheitsdienst und Wohlfahrtspflege Pappelallee 33/35/37, 22089 Hamburg, Deutschland michaela.wolff@tu-dresden.de

\section{Einhaltung ethischer Richtlinien}

Interessenkonflikt. M. Wolff, D. Kitzig, S. Freitag, A. Kozak und A. Nienhaus geben an, dass kein Interessenkonflikt besteht.

Die im Rahmen dieser Studie anonym erhobenen Daten wurden nach den Vorgaben des Bundesdatenschutzgesetzes (BDSG) und des Hamburgischen Datenschutzgesetzes (HmbDSG) ausgewertet und veröffentlicht.

Open Access. Dieser Artikel wird unter der Creative Commons Namensnennung 4.0 International Lizenz veröffentlicht, welche die Nutzung, Vervielfältigung, Bearbeitung, Verbreitung und Wiedergabe in jeglichem Medium und Format erlaubt, sofern Sie den/die ursprünglichen Autor(en) und die Quelle ordnungsgemäßnennen, einen Link zur Creative Commons Lizenz beifügen und angeben, ob Änderungen vorgenommen wurden.

Die in diesem Artikel enthaltenen Bilder und sonstiges Drittmaterial unterliegen ebenfalls der genannten Creative Commons Lizenz, sofern sich aus der Abbildungslegende nichts anderes ergibt. Sofern das betreffende Material nicht unter der genannten Creative Commons Lizenz steht und die betreffende Handlung nicht nach gesetzlichen Vorschriften erlaubt ist, ist für die oben aufgeführten Weiterverwendungen des $\mathrm{Ma}$ terials die Einwilligung des jeweiligen Rechteinhabers einzuholen.

Weitere Details zur Lizenz entnehmen Sie bitte der Lizenzinformation auf http://creativecommons.org/ licenses/by/4.0/deed.de.

\section{Literatur}

1. Amodeo M, Boudot H, Desfray F et al (2004) La coiffure: une enquête de terrain en Côte-d'Or. Doc Méd Trav 99:367-381

2. Caffier G, Steinberg U, Liebers F (1999) Praxisorientiertes Methodeninventar zur Belastungs- und Beanspruchungsbeurteilung im Zusammenhang mit arbeitsbedingten Muskel-Skelett-Erkrankungen. Wirtschaftsverlag NW, Bremerhaven

3. Cohen J (1988) Statistical power analysis for the behavioral sciences. Lawrence Erlbaum, Hillsdale

4. De Smet E, Germeys F (2009) Prevalence of work related upper limb disorders in hairdressers. Work 34:325-330

5. Deschamps F, Langrand J, Lesage FX (2014) Health assessment of self-employed hairdressers in France. J Occup Health 56:157-163

6. Douwes M, Blatter BM, Eikhout SM et al (2001) Onderzoek in het kader van het arboconvenant fysieke belasting bij kappers. TNO Arbeid. www. repository.tudelft.nl/view/tno/uuid\%3Af8f7f62952df-4ae6-85c1-1bdb08f7e14f. Zugegriffen: 31. Mai 2020

7. Foss-Skiftesvik MH, Winther L, Johnsen CR et al (2017) Incidence of skin and respiratory diseases among Danish hairdressing apprentices. Contact Derm 76:160-166

8. Guo HR, Tanaka S, Cameron LL et al (1995) Back pain among workers in the United States: national estimates and workers at high risk. Am J Ind Med 28:591-602

9. Hanvold TN, Wærsted M, Mengshoel AM et al (2013) The effect of work-related sustained trapezius muscle activity on the development of neck and shoulder pain among young adults. Scand JWork Environ Health 39:390-400

10. Hanvold TN, Waersted M, Mengshoel AM et al (2015) Work with prolonged arm elevation as a risk factor for shoulder pain: a longitudinal study among young adults. Appl Ergon 47:43-51

11. Hanvold TN, Waersted M, Mengshoel AM et al (2014) Work with prolonged arm elevation as a risk factor for shoulder pain: a longitudinal study among young adults. Scand J Work Environ Health 40:597-609

12. Kitzig D, Freitag S, Nienhaus A (2015) Muskel-Skelett-Belastungen bei Beschäftigten im Friseurhandwerk. Zbl Arbeitsmed 65:21-27

13. Kitzig D, Hoehne-Hückstädt U, Freitag S et al (2017) Körperhaltungen und Bewegungen bei typischen Friseurtätigkeiten. Zbl Arbeitsmed 67:78-90

14. Kozak A, Wirth T, Verhamme $M$ et al (2019) Musculoskeletal health, work-related risk factors and preventive measures in hairdressing: a scoping review. J Occup Med Toxicol 14:24

15. Kuorinka I, Jonsson B, Kilbom A et al (1987) Standardised Nordic questionnaires for the analysis of musculoskeletal symptoms. Appl Ergon 18:233-237

16. Leino $T$, Tuomi K, Paakkulainen $H$, Klockars $M$ (1999) Health reasons for leaving the profession as determined among Finnish hairdressers in 1980-1995. Int Arch Occup Environ Health 72:56-59

17. Lysdal SH, Søsted H, Andersen KE et al (2011) Hand eczema in hairdressers: a Danish register-based study of the prevalence of hand eczema and its career consequences. Contact Derm 65:151-158

18. Mussi G, Gouveia N (2008) Prevalence of workrelated musculoskeletal disorders in Brazilian hairdressers. Occup Med 58:367-369

19. Nanyan P, Charrada MB (2020) Compensation claims for work-related musculoskeletal disorders among hairdressers in France, 2010-2016. Int J Occup SafErgon 26(4):824-828

20. Roquelaure Y, Ha C, Nicolas G et al (2008) Attributable risk of carpal tunnel syndrome according to industry and occupation in a general population. Arthritis Rheum 59:1341-1348

21. Schneider S, Lipinski S, Schiltenwolf M (2006) Occupations associated with a high risk of selfreported back pain: representative outcomes of a back pain prevalence study in the Federal Republic of Germany. Eur Spine J 15:821-833

22. Veiersted KB, Schulderud Gould K et al (2008) Effect of an intervention addressing working technique on the biomechanical load of the neck and shoulders among hairdressers. Appl Ergon 39:183-190

23. World Health Organization (2000) Obesity: preventing and managing the global epidemic. WHO Obesity Technical Report Series, Bd. 894. WHO, Genf 